\title{
Brain Iron Accumulation Type I Syndrome
}

National Cancer Institute

\section{Source}

National Cancer Institute. Brain Iron Accumulation Type I Syndrome. NCI Thesaurus. Code C8967.

A rare neuroaxonal dystrophy, histologically characterized by axonal spheroids, iron deposition, lewy body (LB)-like intraneuronal inclusions and neurofibrillary tangles. 